\title{
Over- and down-expression mir-29c and mir-21 after chemotherapy and radio- therapy in nasopharyngeal carcinomas and the down-regulating proteins encoding eipstein barr virus and c-Myc.
}

Tirta wardana ${ }^{1 *}$, Cita Herawati ${ }^{2}$, Risky Oktriani ${ }^{3}$, Sumadi Lukman Anwar ${ }^{4}$, Indwiani Astuti $^{5}$, Teguh Aryandono ${ }^{4}$, Sofia Mubarika Haryana ${ }^{6}$

${ }^{1}$ Department of Molecular Biology, Faculty of Medicine, Universitas Gadjah Mada, Indonesia

2Department of Otolaryngology, Dharmais Cancer Hospital, Jakarta Indonesia.

${ }^{3}$ Department of Biochemistry, Faculty of Medicine, Universitas Gadjah Mada, Indonesia

${ }^{4}$ Department of Surgery, Faculty of Medicine, Universitas Gadjah Mada, Indonesia ${ }^{5}$ Department of Pharmacology and Therapy, Faculty of Medicine, Universitas Gadjah Mada, Indonesia

${ }^{6}$ Department of Histology and Cell Biology, Faculty of Medicine, Universitas Gadjah Mada, Indonesia

DOI: http://dx.doi.org/10.19106/JMedScieSup004804201622

\section{ABSTRACT}

Nasopharyngeal carcinoma (NPC) is the type of cancer related to multiple risk factors, including infection by Epstein Barr Virus (EBV). Standard treatment of NPC involves radiotherapy and chemotherapy in local and advanced tumors, while metastatic cases are treated with systemic chemotherapy. However, there is limited data on the causes of tumor recurrence, resistance, and progression. Moreover, the initial symptoms of NPC were often neglected until later enlarged, thus making it difficult to manage. MicroRNA (miRNA) is short molecule with 18-24 nucleotides and functions as protein-expression regulator protein in posttranscription. This study was aimed to determine miRNA expression and its relationship with the incidence of NPC. miR-21 and miR-29c were known to be involved in the development of NPC and resistance. A total of 51 plasma samples and 17 tissue samples were collected from Dharmais Hospital. The samples were taken from 17 untreated patients, 17 treated patients, and 17 healthy participants as control. We examined miRNA, protein of protein EBV (EBNA), and $\mathrm{c}-\mathrm{Myc}$ expression using immunohistochemistry and quantitative polymerase

Corresponding author: tirtawardann@gmail.com 
chain reaction (qPCR). Our study revealed an increased expression of miR-21 and decreased expression of miR-29c in patients with NPC. There was also a correlation between the regulation of expression of miR-21 and c-Myc in the treated group of patients, and decreased expression in patients with complete response (CR) $(4.13 \pm 3.65: 2.74 \pm 3.23 ; p<0.1)$. The parameters tend to increase in patients with partial response (PR) $13.00 \pm 5,86$ compared to 8.77 $\pm 8.43 ; \mathrm{p}<0.5)$, while no significant difference in expression of miR-29c in patients with $\mathrm{CR}$ and PR was detected. We concluded that miRNA might be detected in the plasma of NPC patients, and miR-21 might become a useful biomarker to determine therapeutic outcome in NPC patients.

Keywords: nasopharyngeal cancer; miRNA; biomarker 